\title{
Electronic structure and bonding in skutterudite-type phosphides
}

\author{
Miquel Llunell, Pere Alemany, Santiago Alvarez, and Vladlen P. Zhukov* \\ Departament de Química Física and Departament de Química Inorgànica, Universitat de Barcelona, Diagonal 647, \\ 08028 Barcelona, Spain \\ Andreas Vernes \\ Max-Planck-Institut für Festkörperforschung, Heisenbergstrasse 1, D-7000 Stuttgart 80, Germany
}

(Received 13 October 1995)

\begin{abstract}
The electronic structures of the skutterudite-type phosphides $\mathrm{CoP}_{3}$ and $\mathrm{NiP}_{3}$ have been investigated by means of first-principles linear muffin-tin orbital-atomic sphere approximation band-structure calculations. The presence of $\mathrm{P}_{4}$ rings in the skutterudite structure is of great importance in determining the nature of the electronic bands around the Fermi level, composed mainly of $\pi$-type molecular orbitals of these units. The metallic character found for $\mathrm{NiP}_{3}$ should be ascribed to the phosphorus framework rather than to the metal atoms.
\end{abstract}

The skutterudite (i.e., $\mathrm{CoAs}_{3}$-type) structure is found for a number of binary transition-metal pnictides $M X_{3}$ (Ref. 1), with interesting electronic properties that suggest their use as advanced thermoelectric materials. ${ }^{2,3}$ Except for $\mathrm{NiP}_{3}$, all other known compounds in this family $\left(M X_{3}\right.$ with $M=\mathrm{Co}$, $\mathrm{Rh}$, Ir and $X=\mathrm{P}, \mathrm{As}, \mathrm{Sb})$ are isoelectronic and have been found to behave as diamagnetic $p$-type semiconductors. ${ }^{3-13}$ While some of them seem to have substantial optical band gaps (0.45 and $1.4 \mathrm{eV}$ for $\mathrm{CoP}_{3}$ and $\mathrm{IrSb}_{3}$, respectively), no optical gap is found for $\mathrm{CoAs}_{3}$ and $\mathrm{CoSb}_{3} \cdot{ }^{7} \mathrm{NiP}_{3}$, the only known skutterudite ${ }^{14,15}$ of a group-10 metal, shows metallic behavior with Pauli-type paramagnetism. ${ }^{13}$

Closely related crystal structures are found for the ternary compounds $R M_{4} X_{12}$ ( $R=$ rare-earth metal, $M=\mathrm{Fe}, \mathrm{Ru}, \mathrm{Os}$, and $X=\mathrm{P}, \mathrm{As}, \mathrm{Sb})$. In these, the $M_{4} X_{12}$ subnet adopts the skutterudite structure, ${ }^{16-19}$ while the rare-earth metal atoms occupy the cubic positions that are empty in the binary phases. The physical properties of the ternary phosphides La $M_{4} \mathrm{P}_{12}$ are specially interesting, being superconductors with transition temperatures $T_{c}=4.1,7.2$, and $1.8 \mathrm{~K}$ for $M=\mathrm{Fe}, \mathrm{Ru}$, and $\mathrm{Os},{ }^{20}$ respectively.

Although these compounds show interesting physical properties, relatively little is known about their electronic structure, probably because of the complex crystal structure with a relatively open unit cell. So far, only two bandstructure calculations have been reported for the skutteruditetype compounds. In the first study, ${ }^{21}$ the electronic structures of $\mathrm{LaFe}_{4} \mathrm{P}_{12}$ and related compounds were analyzed by using the extended Hückel tight-binding (EHTB) method. Recently, self-consistent band-structure calculations, using the linear augmented plane-wave method for $\mathrm{IrSb}_{3}, \mathrm{CoAs}_{3}$, and $\mathrm{CoSb}_{3}$, have been published by Singh and Pickett. ${ }^{22}$

In this contribution, we report first-principles bandstructure calculations using the linear muffin-tin orbital method in the atomic sphere approximation (LMTO-ASA) (Refs. 23-25) to explore the band structure of $\mathrm{CoP}_{3}$ and $\mathrm{NiP}_{3}$.

\section{CRYSTAL STRUCTURE}

The nature of the highest occupied bands, responsible for the electronic properties of a material, is strongly dependent on its crystal structure. A detailed knowledge of the crystal structure is, therefore, indispensable before analyzing the details of the electronic structure. In the case of the skutterudite structure (space-group $\operatorname{Im} \overline{3}, n$. 204), ${ }^{26}$ the conventional body-centered-cubic unit cell contains eight $M X_{3}$ units. Metal atoms are located on $8 c$ sites, while the nonmetal ones occupy $24 \mathrm{~g}$ sites. The latter positions depend on two parameters $y$ and $z$ that, together with the lattice parameter $a$, completely define the crystal. ${ }^{27-30}$ A simple way to describe the skutterudite structure is to deduce it as a distortion of the more symmetric $\mathrm{ReO}_{3}$ structure, ${ }^{31,32}$ formed by a simple cubic array of metal atoms octahedrally surrounded by six oxygen atoms. This structure can also be considered as an array of vertex-sharing octahedra [Fig. 1(a)].

The characteristic $X_{4}$ rings in skutterudites can be obtained by displacing four of the nonmetal atoms located on parallel edges of a metal cube to its center, as shown in Fig. 1(b). Since each cube has 12 nonmetal atoms on its edges, the displacement must be done simultaneously in eight neighboring cubes. The new unit cell is thus eight times larger than the original $\mathrm{ReO}_{3}$ unit cell. Since there are not enough nonmetal atoms to make $X_{4}$ rings at the center of each cube, the distortion leaves one out of each four metal cubes empty, as shown in Fig. 2(a). In the resulting skutterudite structure, each metal atom is in an octahedral environ-

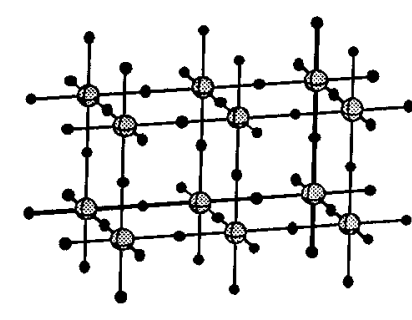

$\mathbf{a}$

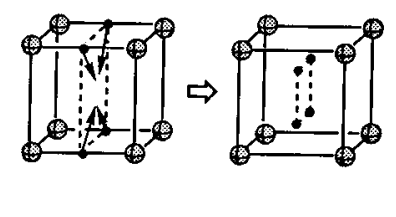

b
FIG. 1. $\mathrm{ReO}_{3}$ crystal structure (a) and distortion that leads to the skutterudite structure (b). Black and gray balls represent $\mathrm{O}$ and $\mathrm{Re}$ atoms, respectively. 


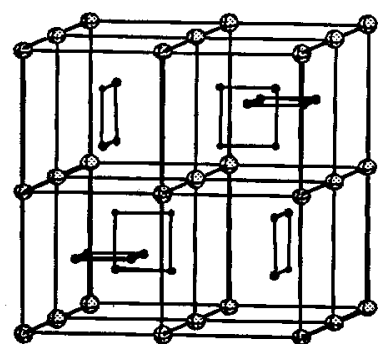

a

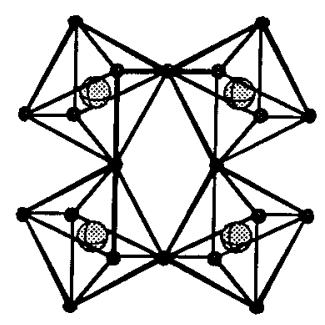

b

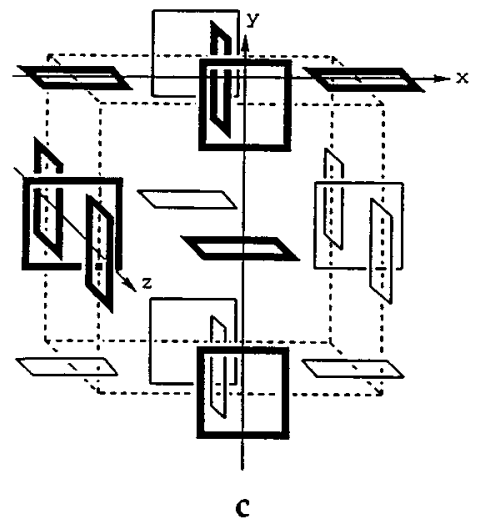

FIG. 2. Skutterudite structure: Unit cell (a), coordination of the metal atoms and array of vertex-sharing octahedra (b), and arrangement of the $X_{4}$ rings in chains (c). Black and gray balls represent nonmetal and metal atoms, respectively. In (c), three mutually orthogonal chains have been highlighted for the sake of clarity.

ment [Fig. 2(b)] and each $M X_{6}$ octahedron is sharing vertices with six neighboring octahedra as in $\mathrm{ReO}_{3}$. The main differences between both structures are that, in the case of skutterudite, the $M X_{6}$ octahedra are distorted (local $D_{3 d}$ symmetry) with their relative orientations in the threedimensional array tilted. The $X_{4}$ rings in the skutterudite structure are arranged in mutually orthogonal linear arrays that run parallel to each crystallographic direction [Fig. 2(c)].

\section{COMPUTATIONAL METHODOLOGY}

Band-structure calculations for $\mathrm{CoP}_{3}$ and $\mathrm{NiP}_{3}$ have been obtained by using the local-density-functional approximation ${ }^{33,34}$ and the scalar-relativistic linear muffin-tin orbital method in the atomic spheres approximation (LMTO-ASA) ${ }^{23-25}$ In this method, the one-electron potential entering the Schrödinger equation is a superposition of overlapping spherical potential wells centered at the atomic positions. A spherical charge density inside the atomic spheres is also assumed. These approximations are specially well suited for highly compact crystal structures. In the case of $\mathrm{CoP}_{3}$ and $\mathrm{NiP}_{3}$ with the relatively open skutterudite structure, the use of only atom-centered spheres would result in substantial errors. To avoid this problem, it has been necessary to introduce interstitial spheres in order to choose sufficiently small radii for the spheres that result in small overlaps between them and at the same time fulfill the usual condition of maximizing the volume of the crystal occupied by the atomic spheres. The location and size of the atomic
TABLE I. Geometrical parameters used for the LMTO-ASAcalculations on $\mathrm{CoP}_{3}$ and $\mathrm{NiP}_{3} . S_{R}$ indicates the radius chosen for each atomic sphere. $E$ represent empty spheres.

\begin{tabular}{lcccc}
\hline \hline Class & $S_{R}(\AA)$ & $x$ & $y$ & $z$ \\
\hline Co, $\mathrm{Ni}(8 c)$ & 1.2748 & 0.2500 & 0.2500 & 0.2500 \\
$\mathrm{P}(24 g)$ & 1.2954 & 0.0000 & 0.3482 & 0.1453 \\
$E_{1}(2 a)$ & 2.0686 & 0.0000 & 0.0000 & 0.0000 \\
$E_{2}(12 e)$ & 0.9938 & 0.0000 & 0.5000 & 0.3524 \\
$E_{3}(24 g)$ & 0.7714 & 0.0000 & 0.1799 & 0.3046 \\
\hline \hline
\end{tabular}

and interstitial spheres has been determined, using the superposition of Hartree atomic potentials. ${ }^{34}$ Application of this procedure to $\mathrm{CoP}_{3}$ and $\mathrm{NiP}_{3}$ results in the sphere positions and radii shown in Table I. Together with the real atoms, our calculations included also 19 empty spheres on each primitive unit cell that, according to symmetry, belong to three nonequivalent classes. The same internal parameters and sphere radii have been used for both compounds. For the cell parameter $a$, the experimentally determined value $(7.073 \AA$ for $\mathrm{CoP}_{3}$ and $7.8192 \AA$ for $\mathrm{NiP}_{3}$ ) has been used in each case.

All calculations were performed within the tight-binding (TB) minimal basis set representation of the LMTO-ASA

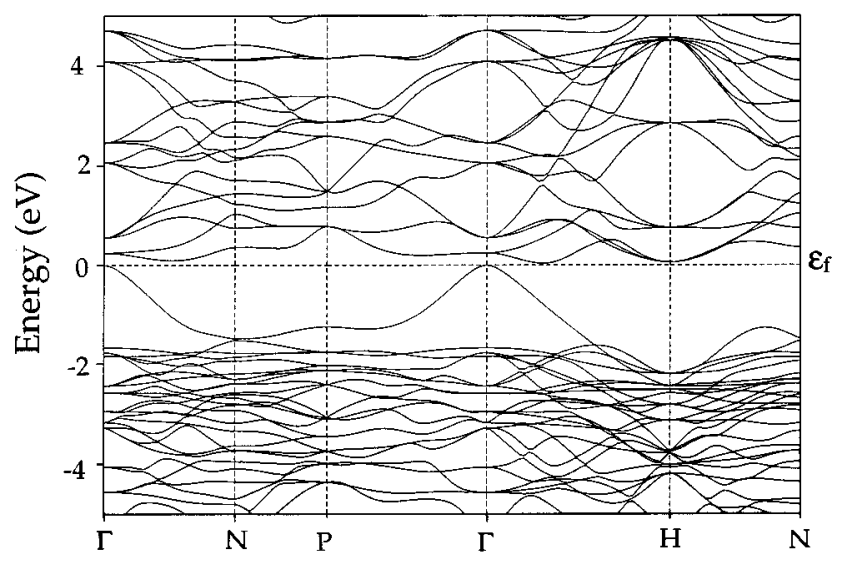

a) $\mathrm{CoP}_{3}$

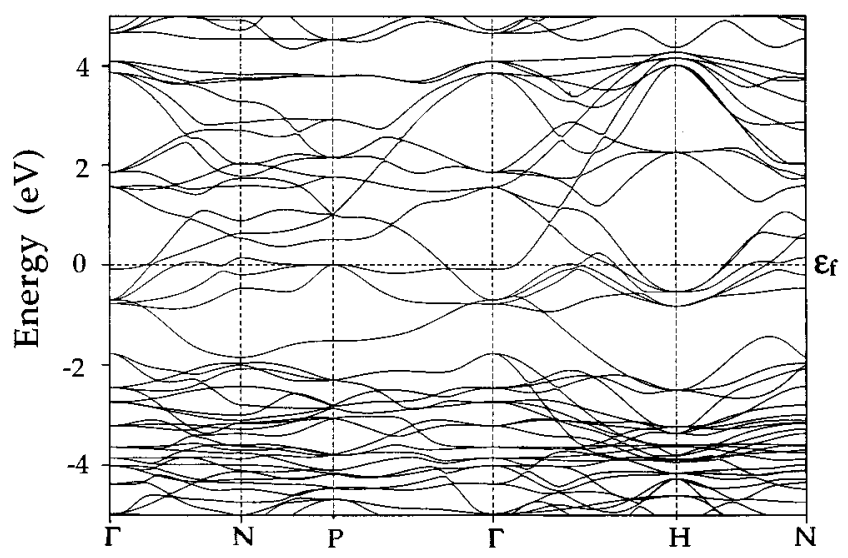

b) $\mathrm{NiP}_{3}$

FIG. 3. LMTO-ASA band structures for $\mathrm{CoP}_{3}$ (a) and $\mathrm{NiP}_{3}$ (b). The horizontal dashed line indicates the Fermi level. 

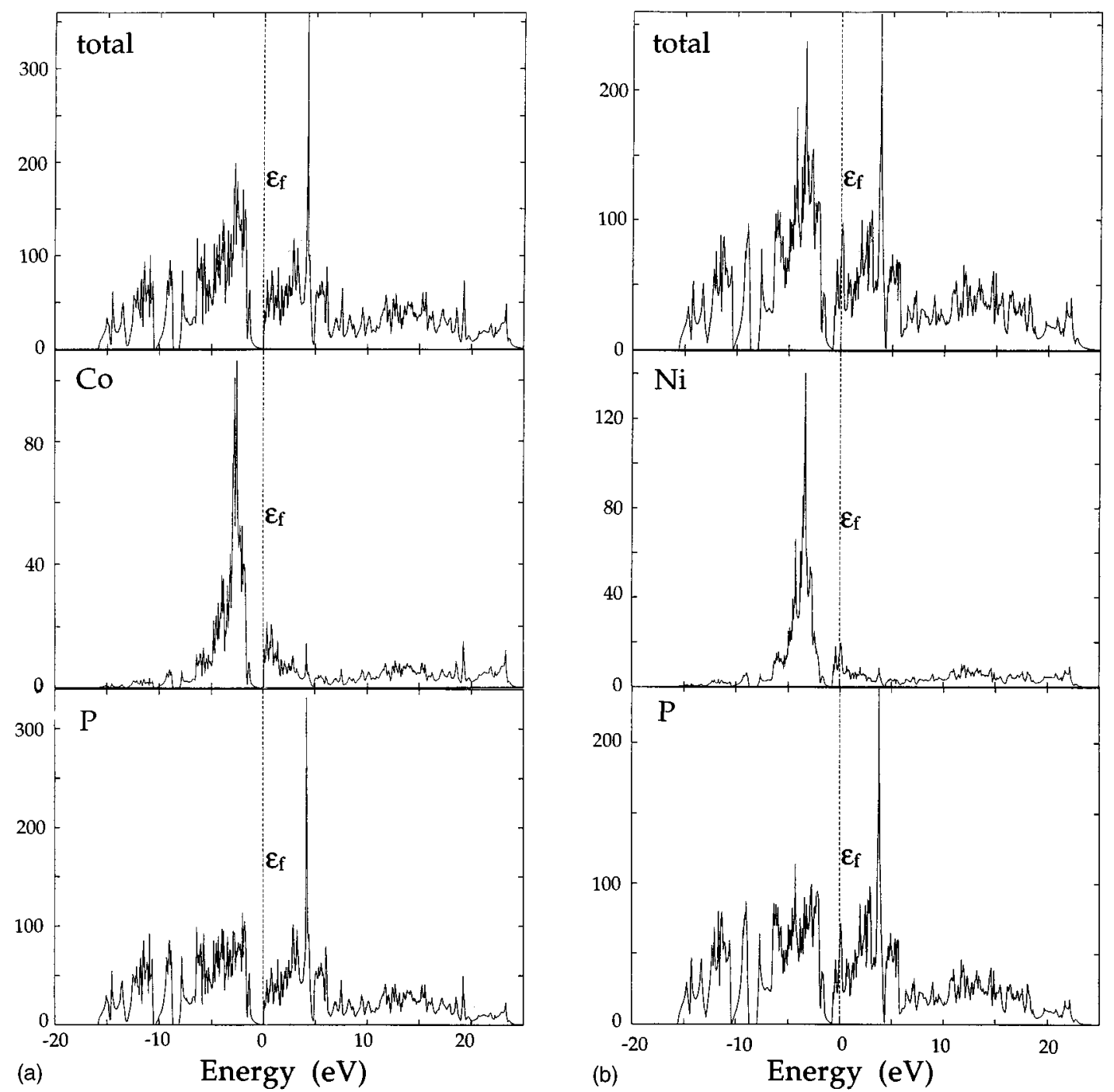

FIG. 4. Total and projected LMTO-ASA electronic density of states (in states/eV cell) for $\mathrm{CoP}_{3}$ (a) and $\mathrm{NiP}_{3}$ (b). The dashed line indicates the Fermi level.

method. ${ }^{25}$ The structure constants were first generated for the short-range TB basis set, but afterwards the transformation to the standard orthonormal basis set has been performed. The down-folding technique ${ }^{25}$ based on Löwdin's perturbation theory $^{35}$ has been applied to this latter set. Integrations in $k$ space were performed using the tetrahedron method ${ }^{36,37}$ with a mesh of 512 points in the irreducible wedge of the first Brillouin zone.

Qualitative features of the electronic structure and bonding in these compounds have been analyzed by using tightbinding band-structure calculations within the extended Hückel framework (EHTB). ${ }^{38-40}$ The parameters used in these calculations are indicated in Ref. 41.

\section{RESULTS AND DISCUSSION}

The calculated (LMTO-ASA) band structures of $\mathrm{CoP}_{3}$ and $\mathrm{NiP}_{3}$ are shown in Figs. 3(a) and 3(b), respectively. The general trends observed in both band-structure diagrams are qualitatively similar to those found by Singh and Pickett ${ }^{22}$ for $\mathrm{IrSb}_{3}, \mathrm{CoSb}_{3}$, and $\mathrm{CoAs}_{3}$. The most important feature in the dispersion diagrams is the more or less well-defined pseudogap that appears just below the Fermi level (see Fig. 4). In $\mathrm{CoP}_{3}$, this pseudogap is crossed by a totally occupied single band that almost reaches the conduction band at the center of the first Brillouin zone. The calculated width of the indirect pseudogap is $1.26 \mathrm{eV}$, that of the indirect band gap (from $\Gamma$ to a point on the $\Gamma-H$ line) is $0.07 \mathrm{eV}$ and that of the direct gap at $\Gamma, 0.28 \mathrm{eV}$. Our calculated direct band gap is somewhat smaller than the value given by Ackermann and Wold ${ }^{7}$ for the optical gap in $\mathrm{CoP}_{3}(0.45 \mathrm{eV})$, probably due to the well-known tendency of the local-density-approximationbased methods to underestimate this property. As already pointed by Singh and Pickett ${ }^{22}$ for $\mathrm{IrSb}_{3}, \mathrm{CoSb}_{3}$, the dispersion of the band in the pseudogap region of $\mathrm{CoP}_{3}$ is remarkable: although it has a parabolic shape in a small region around the $\Gamma$ point, it rapidly becomes linear in energy when leaving the center of the Brillouin zone. This effect is most evident in the $\Gamma-H$ direction, where the crossover between parabolic and linear dispersion occurs for wave vectors of about $5 \%$ the distance to the zone boundary. The transport properties of the hole-doped material should thus be modified by this linear dispersion from those expected for standard semiconductor behavior. 
The dispersion diagram for $\mathrm{NiP}_{3}$, although similar in its rough features to that found for $\mathrm{CoP}_{3}$, shows some distinct features. The larger dispersion of the bands reduces the pseudogap region to approximately $0.57 \mathrm{eV}$. In this case, the band in the pseudogap region penetrates the bottom of the conduction band. In this compound, the metal atoms provide one more electron per formula unit than in $\mathrm{CoP}_{3}$. These electrons occupy the bottom of the conduction band and are responsible for the metallic behavior of $\mathrm{NiP}_{3}$.

Figures 4(a) and 4(b) show the calculated total and projected densities of states (DOS) for both compounds. The pseudogap region with low values for the DOS is clearly visible just below the Fermi level. The phosphorus projection can be roughly divided in three separate regions: the first one ranging from approximately -15 to $-9 \mathrm{eV}$, the second one from $-8 \mathrm{eV}$ to the lower edge of the pseudogap, and the last one starting from the upper edge of the pseudogap. Since these results agree in their main features qualitatively well with those obtained from EHTB calculations (not shown in the figures), this method will be used as a qualitative tool in the analysis of bonding in these materials.

Our EHTB calculations indicate that the lowest region is due basically to the $\sigma$-bonding orbitals of the $\mathrm{P}_{4}$ rings with large phosphorus $3 s$ contributions. The second region is formed mainly by both the nonbonding and the bonding $\pi$-type molecular orbitals of the $\mathrm{P}_{4}$ rings. The third region is due mainly to ring antibonding molecular orbitals, both of $\pi$-type (lowest part) and $\sigma$-type (region above $5 \mathrm{eV}$ ). It is also very important to note that a large contribution of the nonbonding and $\pi$-type bonding orbitals that have the proper spatial orientation to interact strongly with the metal $d$ orbitals is found at the lowest part of this third region. EHTB calculations show for these states an important positive (bonding) overlap population for the P-P bonds.

The projection of the DOS for the metal atoms shows that for both $\mathrm{CoP}_{3}$ and $\mathrm{NiP}_{3}$ the main peak is found in the region directly below the pseudogap. According to these results, the $d$ orbitals of the metal atoms seem to be almost completely filled. This indicates that bonding should be more covalent or metallic than ionic in agreement with the arguments that have been given by some authors based on different experimental evidences. ${ }^{5,10,42-45}$ The most relevant conclusion that can be drawn from these facts is that the bands responsible for the electrical conduction in $\mathrm{NiP}_{3}$ are mainly centered on the phosphorous sublattice. This is clearly visible in our LMTO-ASA calculations, where, the contributions of phosphorous and nickel states to the DOS at the Fermi level are equal to 51.7 and 13.7 states/eV per unit cell, respectively. A more detailed picture of the nature of these states shows that they are mostly formed by nonbonding and $\pi$-type bonding orbitals of the $\mathrm{P}_{4}$ rings. Coupling of these orbitals to neighboring rings in all three crystallographic directions is achieved through interaction with the $s$ and $p$ orbitals of the metal atoms. ${ }^{21}$

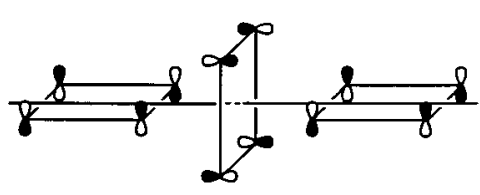

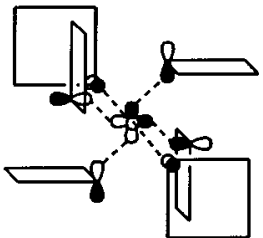

b
FIG. 5. Phosphorous contribution (a) and interaction between phosphorous and metal $p$ orbitals (b) in the highest occupied band for $\mathrm{CoP}_{3}$.

For $\mathrm{CoP}_{3}$, it is also interesting to describe the nature of the single band in the pseudogap region. Our calculations confirm the result obtained earlier by other authors, ${ }^{21}$ indicating that it is basically formed by the $\pi$-type antibonding orbital of the $\mathrm{P}_{4}$ rings shown in Fig. 5(a). The important dispersion of this band is due to mixing of these orbitals belonging to neighboring rings with the metal $p$ orbitals [Fig. 5(b)].

\section{CONCLUDING REMARKS}

First-principles band-structure calculations for $\mathrm{CoP}_{3}$ and $\mathrm{NiP}_{3}$ show that both materials have similar electronic structures with predominantly covalent or metallic bonding. As a consequence, the highest occupied bands are mainly composed of phosphorous-centered orbitals. The electrical properties of phosphorous-rich late transition metal phosphides seem thus to be determined principally by the phosphorous sublattice rather than by the metallic atoms present in the structure. This finding is specially surprising for $\mathrm{NiP}_{3}$, since it has been experimentally found to be a metallic conductor with Pauli paramagnetism. For $d^{6}$ metal skutterudites like $\mathrm{CoP}_{3}$, electrical properties should be determined by the existence of a pseudogap separating the valence and conduction bands. The low density of states in this pseudogap region is provided by a single occupied band that exhibits quasilinear dispersion relations in large parts of the symmetry lines joining $\Gamma$ and the zone boundaries. To confirm whether the highest occupied bands originate largely from the phosphorous or the metal sublattices, it would be interesting to perform a spectroscopic investigation of the valence-band region for this family of compounds.

\section{ACKNOWLEDGMENTS}

The stay of V.Z. in Barcelona was made possible by a sabbatical grant of the Ministerio de Educación y Ciencia (Spain). M.L. thanks CIRIT (Generalitat de Catalunya) for a doctoral grant. The authors are grateful to U. Schönberger for many helpful comments on this work. Financial support was provided by DGICYT through Grant No. PB92-0655.
*On leave from the Institute for Solid State Chemistry, Urals Branch of the Academy of Sciences of Russia, Pervomayskaya 91, GSP-145, Ekaterinburg, Russia.

${ }^{1}$ P. Villars and L. D. Calvert, Pearson's Handbook of Crystallographic Data for Intermetallic Phases (American Society for
Metals, Metals Park, OH, 1986), Vol. 1.

${ }^{2}$ T. Caillat, A. Borshchevsky, and J. P. Fleurial, in Proceedings of the Eleventh International Conference on Thermoelectrics, edited by K. R. Rao (University of Texas at Arlington Press, Arlington, TX, 1993). 
${ }^{3}$ G. A. Slack and V. G. Tsoukala, J. Appl. Phys. 76, 1165 (1994).

${ }^{4}$ F. Hulliger, Helv. Phys. Acta 34, 782 (1961).

${ }^{5}$ A. Kjekshus and G. Pedersen, Acta Crystallogr. 14, 1065 (1961).

${ }^{6}$ C. M. Pleass and R. D. Heyding, Can. J. Chem. 40, 590 (1962).

${ }^{7}$ J. Ackermann and A. Wold, J. Phys. Chem. Solids 38, 1013 (1977).

${ }^{8}$ J. P. Odile, S. Soled, C. A. Castro, and A. Wold, Inorg. Chem. 17, 283 (1978).

${ }^{9}$ H. D. Lutz and G. Kliche, Z. Anorg. Allg. Chem. 480, 105 (1981).

${ }^{10}$ H. D. Lutz and G. Kliche, Phys. Status Solidi B 112, 549 (1982).

${ }^{11}$ G. Kliche and W. Bauhofer, J. Phys. Chem. Solids 49, 267 (1988)

${ }^{12}$ G. Kliche, Solid State Commun. 80, 73 (1991).

${ }^{13}$ K. Zeppenfeld and W. Jeitschko, J. Phys. Chem. Solids 54, 1527 (1993).

${ }^{14}$ Although the synthesis of $\mathrm{PdP}_{3}$ has been reported (see Ref. 15), no reliable crystal structure has been obtained for this compound to the best of our knowledge.

${ }^{15}$ S. Rundqvist, Nature (London) 185, 31 (1960).

${ }^{16}$ W. Jeitschko and D. J. Braun, Acta Crystallogr. Sec. B 33, 3401 (1977).

${ }^{17}$ D. J. Braun and W. Jeitschko, J. Solid State Chem. 32, 357 (1980).

${ }^{18}$ D. J. Braun and W. Jeitschko, J. Less-Common Met. 72, 147 (1980).

${ }^{19}$ D. J. Braun and W. Jeitschko, J. Less-Common Met. 76, 33 (1980).

${ }^{20}$ L. E. DeLong and G. P. Meisner, Solid State Commun. 53, 119 (1985).

${ }^{21}$ D. Jung, M.-H. Whangbo, and S. Alvarez, Inorg. Chem. 29, 2252 (1990).

${ }^{22}$ D. J. Singh and W. E. Pickett, Phys. Rev. B 50, 11235 (1994).

${ }^{23}$ O. K. Andersen and O. Jepsen, Phys. Rev. Lett. 53, 2571 (1984).

${ }^{24}$ O. K. Andersen, Z. Pawlowska, and O. Jepsen, Phys. Rev. B 34, 5253 (1986).

${ }^{25}$ O. K. Andersen, O. Jepsen, and D. Glotzel, in Highlights of Condensed-Matter Theory, edited by F. Bassani, F. Fumi, and M. P. Tosi (North-Holland, New York, 1985).

${ }^{26}$ I. Z. Oftedal, Kristallografiya A 66, 517 (1928).
${ }^{27}$ S. Rundqvist and N.-O. Ersson, Ark. Kemi 30, 103 (1968).

${ }^{28}$ A. Kjekshus and T. Rakke, Acta Chem. Scand. A 28, 99 (1974).

${ }^{29}$ N. Mandel and J. Donohue, Acta Crystallogr. Sec. B 27, 2288 (1971).

${ }^{30}$ R. T. M. Dobbe, W. J. Lustenhouwer, M. A. Zakrzewski, K. Goubitz, J. Fraanje, and H. Schenk, Can. Mineral. 32, 179 (1994).

${ }^{31}$ K. Meisel, Z. Anorg. Allg. Chem. 207, 121 (1932).

${ }^{32}$ T.-S. Chang and P. Trucano, J. Appl. Crystallogr. 11, 286 (1978).

${ }^{33}$ U. von Barth and L. Hedin, J. Phys. C 4, 2064 (1971).

${ }^{34}$ G. Krier, O. K. Andersen, and O. Jepsen (unpublished).

${ }^{35}$ P.-O. Löwdin, J. Chem. Phys. 19, 1396 (1951).

${ }^{36}$ O. Jepsen and O. K. Andersen, Solid State Commun. 9, 1763 (1971).

${ }^{37}$ P. Blöchl, O. Jepsen, and O. K. Andersen, Phys. Rev. B 49, 16223 (1994).

${ }^{38}$ R. Hoffmann, J. Chem. Phys. 39, 1397 (1963).

${ }^{39}$ M.-H. Whangbo and R. Hoffmann, J. Am. Chem. Soc. 100, 6093 (1978).

${ }^{40}$ M.-H. Whangbo, R. Hoffmann, and R. B. Woodward, Proc. R. Soc. London Ser. A 366, 23 (1979).

${ }^{41}$ The exponents $(\zeta)$ and the valence shell ionization potentials $\left(H_{i i}\right.$ in $\mathrm{eV}$ ) used in the EHTB calculations were chosen as follows: 1.81 and -18.40 for $\mathrm{P} 3 s ; 1.45$ and -9.80 for $\mathrm{P} 3 p ; 2.00$ and -9.21 for $\mathrm{Co} 4 s ; 2.00$ and -5.29 for $\operatorname{Co} 4 p ; 1.83$ and -9.17 for $\mathrm{Ni} 4 s ; 1.13$ and -5.15 for $\mathrm{Ni} 4 p . H_{i i}$ values for Co and $\mathrm{Ni} 3 d$ were set equal to -13.18 and $-13.49 \mathrm{eV}$, respectively. A linear combination of two Slater-type orbitals of exponents $\zeta_{1}=5.55$, $\zeta_{2}=2.10$ and $\zeta_{1}=5.75, \zeta_{2}=2.00$ with weighting coefficients $c_{1}=0.568$ and $c_{2}=0.606$ and $c_{1}=0.5683$ and $c_{2}=0.6292$ were used to represent the $3 d$ orbitals of $\mathrm{Co}$ and $\mathrm{Ni}$, respectively.

${ }^{42}$ T. Rosenqvist, Acta Metall. 1, 761 (1953).

${ }^{43}$ A. Kjekshus, D. G. Nicholson, and T. Rakke, Acta Chem. Scand. 27, 1315 (1973).

${ }^{44}$ O. K. Andersen, A. V. Postnikov, and S. Y. Savrasov, in Application of Multiple Scattering Theory to Materials Science, edited by W. H. Butler, P. H. Dederichs, A. Gonis, and R. Weaver, MRS Symposia Proceedings No. 253 (Materials Research Society, Pittsburgh, 1992), p. 27.

${ }^{45}$ J. H. Ammeter, H. B. Bürgi, J. C. Thibeault, and R. Hoffmann, J. Am. Chem. Soc. 100, 3686 (1978). 